\title{
Accuracy of Hybrid Lattice Boltzmann/Finite Difference Schemes for Reaction-Diffusion Systems
}

\author{
Pieter Van Leemput \\ Christophe Vandekerckhove \\ Wim Vanroose \\ Dirk Roose
}

Report TW 476, November 2006

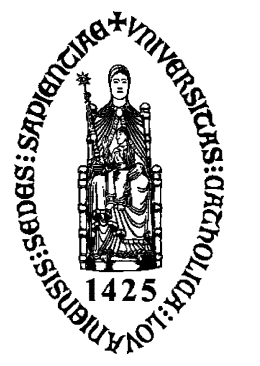

\section{Katholieke Universiteit Leuven}

Department of Computer Science

Celestijnenlaan 200A - B-3001 Heverlee (Belgium) 


\title{
Accuracy of Hybrid Lattice Boltzmann/Finite Difference Schemes for Reaction-Diffusion Systems
}

\author{
Pieter Van Leemput \\ Christophe Vandekerckhove \\ Wim Vanroose \\ Dirk Roose \\ Report TW 476, November 2006
}

Department of Computer Science, K.U.Leuven

\begin{abstract}
In this article we construct a hybrid model by spatially coupling a lattice Boltzmann model (LBM) to a finite difference discretization of the partial differential equation (PDE) for reaction-diffusion systems. Because the LBM has more variables (the particle distribution functions) than the PDE (only the particle density), we have a one-to-many mapping problem from the PDE to the LBM domain at the interface. We perform this mapping using either results from the Chapman-Enskog expansion or a point-wise iterative scheme that approximates these analytical relations numerically. Most importantly, we show that the global spatial discretization error of the hybrid model is one order less accurate than the local error made at the interface. We derive closed expressions for the spatial discretization error at steady state and verify them numerically for several examples on the one-dimensional domain.
\end{abstract}

Keywords : accuracy, spatial discretization error, hybrid model, lattice Boltzmann, finite difference, spatial coupling, constrained runs.

AMS(MOS) Classification : 76M28, 65M99, 35K57. 


\title{
Accuracy of Hybrid Lattice Boltzmann/Finite Difference Schemes for Reaction-Diffusion Systems
}

\author{
P. Van Leemput, C. Vandekerckhove, W. Vanroose and D. Roose \\ Department of Computer Science, Katholieke Universiteit Leuven, \\ Celestijnenlaan 200A, B-3001 Heverlee, Belgium \\ e-mail: pieter.vanleemput@cs.kuleuven.be
}

16th November 2006

\begin{abstract}
In this article we construct a hybrid model by spatially coupling a lattice Boltzmann model (LBM) to a finite difference discretization of the partial differential equation (PDE) for reaction-diffusion systems. Because the LBM has more variables (the particle distribution functions) than the PDE (only the particle density), we have a one-to-many mapping problem from the PDE to the LBM domain at the interface. We perform this mapping using either results from the Chapman-Enskog expansion or a point-wise iterative scheme that approximates these analytical relations numerically. Most importantly, we show that the global spatial discretization error of the hybrid model is one order less accurate than the local error made at the interface. We derive closed expressions for the spatial discretization error at steady state and verify them numerically for several examples on the one-dimensional domain.

Keywords: accuracy, spatial discretization error, hybrid model, lattice Boltz-
\end{abstract} mann, finite difference, spatial coupling, constrained runs.

AMS(MOS) Classification: 76M28, 65M99, 35K57.

\section{Introduction}

When given the task of simulating a dynamical system, one has a variety of models to choose from, each operating on a different level of abstraction. It depends both on the complexity of particle interactions (atoms or molecules) and the corresponding level of detail required (either for correct simulation or the detail of interest to the user) which model is appropriate. On the microscopic level, one has molecular dynamics methods which monitor the individual particles. Kinetic Monte Carlo methods provide a higher level of abstraction by modeling the statistics of the particle interactions. Even more coarse-grained are mesoscopic or pseudo-particle models, like lattice Boltzmann models (LBMs). They describe the system's evolution through generalized distributions of particles which are limited to move on a grid with particular velocities only. Finally, on the macroscopic or continuum level, one has appropriately discretized ordinary or partial differential equations (ODEs or PDEs) in terms of a few low order moments (observables like density, flow velocity, pressure, ...).

The choice for a particular model depends on several criteria. Macroscopic-level models, like PDEs, typically have a smaller dimensional state space and in general allow large time steps during simulation. When a macroscopic description for the system's evolution can not be derived in terms of the macroscopic variables only, i.e. when the macroscopic model does not close, a lower level model describing the 
same physics in more detail should be used. Because of their notion of, albeit idealized, particles, mesoscopic models like LBMs allow the incorporation of complicated physics in a more bottom-up way than macroscopic models. Also, the LBM particle distribution functions contain information on the spatial derivatives of the macroscopic variables because they are linked to the particle velocities. Finally, they can treat irregular domain boundaries in a natural way. However, these models typically require more variables and smaller time steps. Similar advantages apply to microscopic models, but simulation with these models can be very expensive and often becomes prohibitive.

Sometimes, the level of detail required to model a physical system changes from region to region and different models have to be used on different parts of the domain. Many hybrid methods which couple a particle-based to a continuum model have already been well developed, see e.g. [1, 4, 14, 17, 20, 25] and references therein. At the interface between the regions, there will be a mismatch in the kind (and number) of variables used by the different models. There, the variables have to mapped to one another.

In this article, we analyze hybrid models obtained by spatially coupling a LBM and the finite difference discretization (FD) of a PDE describing a reaction-diffusion system in different regions of the spatial domain. We are particularly interested in the spatial discretization error of the hybrid model. In [2, 3, 24], Albuquerque et al. reported a second order discretization error in space (just like both the discretized PDE and LBM on the full domain). We will study this claim further and derive closed expressions for the error at steady state for some basic problems.

For analysis purposes, we only consider one-dimensional reaction-diffusion problems. For this setup, the LBM has three times as much variables (the particle distribution functions) as the PDE, which evolves only the particle density. At the interface, densities are obtained as the zeroth order velocity moments of the distribution functions. However, the inverse mapping from the PDE to the LBM domain is not univocal. Note that the same one-to-many problem also occurs when initializing a LBM from macroscopic initial data $[28,35,36]$.

We discuss two methods to solve the mapping problem at the interface. The first, analytical method was introduced by Albuquerque et al. [2, 3]. They used the Chapman-Enskog expansion to write the missing distribution functions at the hybrid interface as a first order functional of the density variable. We will use the same concept but a different implementation. The second method is a point-wise iterative scheme that approximates the Chapman-Enskog relations numerically. It is useful when these functionals are not available or difficult to obtain analytically. Examples are the constrained runs scheme developed by Gear and Kevrekidis $[19,18]$ in the context of equation-free computing and the scheme developed by Mei et al. [28, 8] in the context of LBM initialization. Here we use the former. The constrained runs scheme performs a series of short microscopic (here LBM) simulations and resets the lowest order velocity moment (density) to its initial value while leaving the higher order moments unchanged. We showed in [36] that the application of the scheme to the LBM considered here, produces a numerical approximation of the analytical Chapman-Enskog relations that is correct up to first order.

The work described in this article is a step in the development of efficient methods for hybrid LBM/PDE coupling. In the discussion, we have made some simplifying assumptions. First, we use the same time step and grid spacing for both the PDE and LBM. Using different space-time grids on the sub-domains is an interesting topic of further research. For the time dependent problem, we refer to grid refinement techniques like adaptive mesh refinement (AMR) [5] for the PDE and the different approaches in $[31,15,16,23]$ for the LBM and to certain implicit $[9,26,33]$ or outer explicit [38] time discretization schemes. For the time independent problem, ideas from multigrid [34] for the PDE and [27, 32] for the 
LBM or iterative methods, like the preconditioned Generalized Method of Residuals (GMRES) [6, 7] or the biconjugate gradient algorithm [39] could be used. Second, we limit ourself to one-dimensional diffusive problems for analysis purposes. Albuquerque, Latt and coworkers already used the Chapman-Enskog based coupling method for two-dimensional diffusion [3] and Poiseuille flow [24]. Finally, we work with LBM and PDE models which are equivalent in the macroscopic limit. I.e. since the macroscopic solution profile is sufficiently smooth on the whole domain, we could have used either the PDE or LBM on the full domain. The coupling of a PDE and a LBM describing distinct dynamical behavior will be discussed elsewhere.

This article is organized as follows. In Sect. 2, we introduce the PDE and LBM for one-dimensional reaction-diffusion systems and the relations between the two. In Sect. 3, we discuss the constrained runs scheme. Section 4 presents the different coupling schemes. We derive expressions for the steady state discretization error in Sect. 5. The theoretical results are verified numerically in Sect. 6. Finally, in Sect. 7, we summarize the main conclusions of the paper.

\section{Reaction-Diffusion Models}

In this section, we present the macroscopic PDE (Sect. 2.1) and mesoscopic LBM with BGK collision model (Sect. 2.2) for one-dimensional reaction-diffusion systems. In Sect. 2.3 we describe the relations between the two.

\subsection{Partial Differential Equation (PDE)}

For diffusive systems, the macroscopic variable describing the system's evolution is the particle density (concentration) $\rho(x, t)$ as a function of space $x$ and time $t$. The corresponding PDE is given by

$$
\frac{\partial \rho(x, t)}{\partial t}=D \frac{\partial^{2} \rho(x, t)}{\partial x^{2}}+F(\rho(x, t))
$$

where $D$ is the diffusion coefficient and $F(\rho(x, t))$ a macroscopic reaction force term which depends on $\rho(x, t)$ only. This continuous equation is discretized with finite differences (FD) (explicit forward difference in time and central difference in space) to obtain

$$
\rho(x, t+\Delta t)=\rho(x, t)+\frac{\Delta t D}{\Delta x^{2}}(\rho(x+\Delta x, t)-2 \rho(x, t)+\rho(x-\Delta x, t))+\Delta t F(\rho(x, t))
$$

with $\Delta x$ and $\Delta t$ the corresponding space and time step. It is well known that the discretization error for (2) is first order in time and second order in space (to leading order: $\left.-\left(\Delta x^{2} / 12\right) \partial^{4} \rho(x, t) / \partial x^{4}\right)$. Note that this discretization typically entails a stability condition of the form $\Delta t \leq C \Delta x^{2}$, e.g. for pure diffusion, the constant $C$ equals $1 /(2 D)$.

\subsection{Lattice Boltzmann Model (LBM)}

Lattice Boltzmann models (LBM) [10, 11, 29] describe the evolution of particle distribution functions $f_{i}(x, t)$ discretized in space $x$, time $t$ and velocity $v_{i}$. These distributions are defined on a space-time lattice with grid spacing $\Delta x$ in space and $\Delta t$ in time. On a one-dimensional domain, only three values are considered for the velocity (D1Q3 model):

$$
v_{i}=c_{i} \frac{\Delta x}{\Delta t}, \quad c_{i}=i \in\{-1,0,1\}
$$


with $c_{i}$ the dimensionless lattice velocity.

The lattice Boltzmann equation (LBE) describing the evolution of the distribution functions is defined as

$$
f_{i}\left(x+c_{i} \Delta x, t+\Delta t\right)=(1-\omega) f_{i}(x, t)+\omega f_{i}^{e q}(x, t)+R_{i}(x, t)
$$

for $i \in\{-1,0,1\}$. The right hand side of (4) updates the values $f_{i}(x, t)$ to postcollision values $f_{i}^{\star}\left(x, t^{\star}\right)$ (with $t<t^{\star}<t+\Delta t$ ). Afterwards, these values propagate to a neighboring lattice site according to their velocity direction (left hand side of (4)). Diffusive collisions are modeled by the Bhatnagar-Gross-Krook (BGK) collision term $-\omega\left(f_{i}(x, t)-f_{i}^{e q}(x, t)\right)$ in (4) as a relaxation to a local diffusive equilibrium $f_{i}^{e q}(x, t)$ with relaxation coefficient $\omega[30,37]$, while reactions are modeled by the term $R_{i}(x, t)[30,13]$ as

$$
f_{i}^{e q}(x, t)=\frac{1}{3} \rho(x, t), \quad \omega=\frac{2}{1+3 D \frac{\Delta t}{\Delta x^{2}}}, \quad R_{i}(x, t)=\frac{\Delta t}{3} F(\rho(x, t)),
$$

with $D$ and $F(\rho(x, t))$ defined in (1). Here, it is assumed that reactions occur at the local diffusive equilibrium [12]. As shown in [22], the LBM discretization error is of second order in space.

The particle density $\rho(x, t)$ is defined as the zeroth order velocity moment of the distribution functions

$$
\rho(x, t)=\sum_{i=-1}^{1} f_{i}(x, t)=\sum_{i=-1}^{1} f_{i}^{e q}(x, t),
$$

where the second equality expresses that the BGK diffusive collisions locally conserve density (compare (5)). When we define momentum $\phi$ and (kinetic) energy $\xi$ as the first respectively ( $1 / 2$ times the) second order velocity moment, the LBM state at time $t$ and position $x$ can also be described in terms of these moments. Using $\mathbf{f}=\left[\begin{array}{lll}f_{-1} & f_{0} & f_{1}\end{array}\right]^{\prime}$ and $\varrho=\left[\begin{array}{l}\rho \\ \xi\end{array}\right]^{\prime}$, one can easily switch from one representation to the other by

$$
\varrho=\left[\begin{array}{c}
\rho \\
\phi \\
\xi
\end{array}\right]=\left[\begin{array}{rrr}
1 & 1 & 1 \\
-1 & 0 & 1 \\
\frac{1}{2} & 0 & \frac{1}{2}
\end{array}\right]\left[\begin{array}{c}
f_{-1} \\
f_{0} \\
f_{1}
\end{array}\right]=M \mathbf{f}
$$

and vice versa $\mathbf{f}=M^{-1} \varrho$ (one-to-one relationship). Note that we refer to the higher order velocity moments $\phi$ and $\xi$ as "momentum" and "energy" although these are non-conserved quantities in a diffusive system.

\subsection{Relations between LBM and PDE}

When the system's solution varies slowly on a macroscopic length and time scale, both the LBM and PDE can be used to describe its evolution. Under this condition, the LBM from Sect. 2.2 can be reduced to the PDE from Sect. 2.1 using a multiscale Chapman-Enskog expansion [10, 11]. For the model problem discussed here, the details are given in [37]. In this expansion, the distribution functions are written as

$$
f_{i}(x, t)=f_{i}^{[0]}(x, t)+f_{i}^{[1]}(x, t)+f_{i}^{[2]}(x, t)+\ldots
$$

in terms of increasingly higher order contributions. The zeroth and first order contribution $f_{i}^{[0]}$ and $f_{i}^{[1]}$ are

$$
f_{i}^{[0]}=f_{i}^{e q}=\frac{1}{3} \rho, \quad f_{i}^{[1]}=-\frac{\Delta x}{\omega} c_{i} \cdot \nabla f_{i}^{[0]}=-\frac{c_{i} \Delta x}{3 \omega} \frac{\partial \rho}{\partial x},
$$




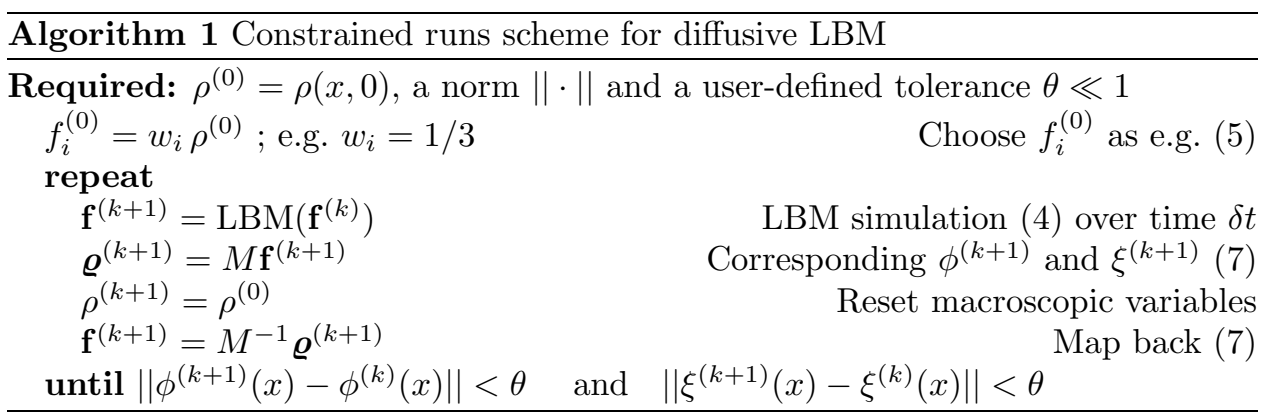

while the second order contribution $f_{i}^{[2]}$ can be derived as

$$
f_{i}^{[2]}=-\frac{\Delta t}{6 \omega}\left(3 c_{i}^{2}-2\right)\left(F(\rho)-\frac{\partial \rho}{\partial t}\right)=-\frac{\Delta x^{2}}{18 \omega^{2}}(\omega-2)\left(3 c_{i}^{2}-2\right) \frac{\partial^{2} \rho}{\partial x^{2}} .
$$

These so-called slaving relations are functionals of the macroscopic slow (density) variable only. The equivalent PDE (1) is obtained by summing (10) over all velocities, where $\sum_{i} f_{i}^{[2]}=0$ (sum up (8) and use (6)). Because of the discrete LBE propagation, the continuous spatial derivatives in (9)-(10) can be seen as a shorthand notation for the appropriate central finite difference scheme corresponding to the LBM (D1Q3) stencil and must be discretized accordingly (see also [21, 36]).

\section{Constrained Runs Scheme}

Given only the density values on the domain, the full state of the LBM is described up to second order by the slaving relations (9)-(10). When such relations are unavailable or difficult to obtain analytically due to e.g. complex local interaction force terms, the constrained runs (CR) scheme [19, 18] can be used to approximate these relations numerically. Algorithm 1 describes the CR scheme for the LBM from Sect. 2.2. For a given density profile $\rho^{(0)}$, an initial guess for $f_{i}^{(0)}$ would be e.g. the BGK equilibrium distribution (5). The LBM is then repeatedly used to evolve the state for a short time $\delta t$. After each such simulation, the lowest moment of the distribution functions is reset to the initial density profile.

The CR scheme can be defined as a map

$$
\varrho^{(k+1)}=\mathcal{C}_{\delta t}\left(\varrho^{(k)}\right) ; \quad k=0,1,2, \ldots, K
$$

on the state vector $\varrho^{(k)}=\left[\rho^{(0)} \phi^{(k)} \xi^{(k)}\right]^{\prime}$; with $k$ the iteration number. Since $\rho^{(k+1)}$ is reset to $\rho^{(0)}$ after each step, the map effectively iterates on the higher order moments $\phi$ and $\xi$ to obtain the fixed point.

In [36] we analyzed the use of the CR scheme for the initialization of the LBM for one-dimensional reaction-diffusion problems from Sect. 2.2. We proved that the scheme is unconditionally stable and convergent with asymptotic convergence factor $|1-\omega|$. The fixed point in moment space $\left\{\left(\rho^{(0)}\right), \tilde{\phi}, \tilde{\xi}\right\}$ can be written in terms of distribution functions as $\left\{\tilde{f}_{-1}, \tilde{f}_{0}, \tilde{f}_{1}\right\}$ using $(7)$ and is given by $[36,37]$

$$
\tilde{f}_{i}=\frac{1}{3} \rho^{(0)}-\frac{c_{i} \Delta x}{3 \omega} \frac{\partial \rho^{(0)}}{\partial x}-\frac{\Delta t}{6 \omega}\left(3 c_{i}^{2}-2\right)\left(F\left(\rho^{(0)}\right)-3 \frac{\left(\tilde{\rho}-\rho^{(0)}\right)}{\Delta t}\right)
$$

with $\tilde{\rho}$ the internal simulated-upon density corresponding to $\{\tilde{\phi}, \tilde{\xi}\}$ (before the final reset to $\left.\rho^{(0)}\right)$. When comparing to $(9)-(10)$, we see that $(11)$ is a first order correct approximation of the slaved state. The difference $\tilde{\rho}-\rho^{(0)}$ is a measure for the error. To keep this error as small as possible, we choose $\delta t=\Delta t$ (one LBM time step). 


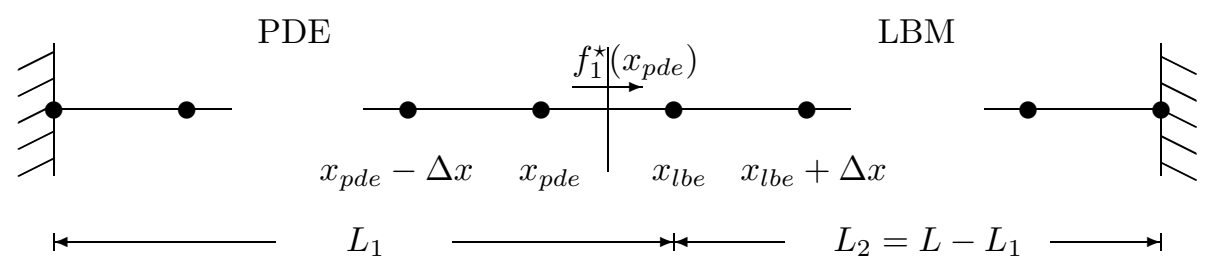

Figure 1: Spatial coupling of the PDE (left) and LBM (right) on a one-dimensional domain. The interface lies in between two lattice sites.

\section{Hybrid Spatial Coupling}

In this section, we describe how to couple the PDE and LBM from Sect. 2. In our setup shown in Fig. 1, the one-dimensional domain with length $L$ is split into two non-overlapping sublattices. An alternative using one overlapping lattice site is presented in $[2,3]$ and will be briefly discussed in Sect. 6. The PDE is applied to the left sublattice and the LBM to the right sublattice. The coordinate of the first LBM lattice point is defined as $x_{l b e}=L_{1}$, such that the interface lies in between $x_{p d e}$ and $x_{l b e}$ at $L_{1}-\Delta x / 2$. For analysis purposes, we use the same lattice spacing $\Delta x$ and time step $\Delta t$ for the PDE and LBM, i.e. the simplest coupled space-time lattice.

Since the PDE and LBM use a different set of variables, namely $\rho$ versus $\mathbf{f}=$ $\left[f_{-1} f_{0} f_{1}\right]^{\prime}$, information should be exchanged carefully at the interface during time simulation. To evolve the PDE in $x_{p d e}$, the value $\rho\left(x_{l b e}, t\right)=\rho\left(x_{p d e}+\Delta x, t\right)$ is needed in (2). This value is computed from the LBM variables $f_{i}\left(x_{l b e}, t\right)$ using (6). The inverse problem is more difficult. We need to map a single density value onto three corresponding distributions, i.e. $\rho\left(x_{p d e}, t\right) \mapsto f_{i}\left(x_{p d e}, t\right)$ for $i \in\{-1,0,1\}$. Since (6) should hold, this leaves two degrees of freedom. In fact, we need only the value of $f_{1}\left(x_{p d e}, t\right)$ that propagates into the LBM subdomain (see Fig. 1) to evolve the LBE (4) in $x_{l b e}$ from $t$ to $t+\Delta t$. We will use the analytical slaving relations (9)-(10) or the numerical approximation (11) by the CR scheme to derive the distributions at the interface. In the next sections we discuss both strategies.

\subsection{Implementation using Chapman-Enskog Relations}

The key observation is that the PDE (2) simulates "directly" from $t$ to $t+\Delta t$, while the LBE (4) executes in two phases: first, collisions and reactions to go from $t$ to $t^{\star}$ and secondly, propagation of the post-collision distributions $f_{i}^{\star}$ to get from $t^{\star}$ to $t+\Delta t$. Thus we actually need the post-collision value $f_{1}^{\star}\left(x_{p d e}, t^{\star}\right)$ rather than the value $f_{1}\left(x_{p d e}, t\right)$.

We first reconstruct $f_{1}\left(x_{p d e}, t\right)$ from the PDE density using the Chapman-Enskog relations up to first order $(9)$ as in $[2,3]$

$$
\begin{aligned}
f_{1}\left(x_{p d e}, t\right) & =f_{1}^{[0]}\left(x_{p d e}, t\right)+f_{1}^{[1]}\left(x_{p d e}, t\right) \\
& =\frac{1}{3} \rho\left(x_{p d e}, t\right)-\frac{\Delta x}{3 \omega} \frac{\rho\left(x_{l b e}, t\right)-\rho\left(x_{p d e}-\Delta x, t\right)}{2 \Delta x},
\end{aligned}
$$

where the continuous derivative $\partial \rho\left(x_{p d e}, t\right) / \partial x$ is discretized with central differences (cf. Sect. 2.3). The value $\rho\left(x_{l b e}, t\right)$ is obtained from the LBM domain using (6).

Afterwards, the corresponding post-collision value $f_{1}^{\star}\left(x_{p d e}, t^{\star}\right)$ is computed from (12) using the LBE:

$$
f_{1}^{\star}\left(x_{p d e}, t^{\star}\right)=(1-\omega) f_{1}\left(x_{p d e}, t\right)+\frac{\omega}{3} \rho\left(x_{p d e}, t\right)+\frac{\Delta t}{3} F\left(\rho\left(x_{p d e}, t\right)\right) .
$$


Finally, it is this value that is propagated to $x_{l b e}$, i.e.

$$
f_{1}\left(x_{l b e}, t+\Delta t\right)=f_{1}^{\star}\left(x_{p d e}, t^{\star}\right)
$$

From now on, we refer to (12)-(14) as the CE-1 coupling scheme. Note that the outgoing post-collision value $f_{-1}^{\star}\left(x_{l b e}, t^{\star}\right)$ that enters the PDE domain is never used.

Below, we define variants of the above coupling scheme using additional or fewer terms in the Chapman-Enskog expansion. By adding the term $f_{1}^{[2]}\left(x_{p d e}, t\right)(10)$ (after appropriate discretization with central finite differences), i.e.

$$
f_{1}^{[2]}\left(x_{p d e}, t\right)=-\frac{(\omega-2)}{18 \omega^{2}}\left(\rho\left(x_{l b e}, t\right)-2 \rho\left(x_{p d e}, t\right)+\rho\left(x_{p d e}-\Delta x, t\right)\right),
$$

to (12), we have a second order local coupling scheme (denoted by CE-2). For reasons of comparison only, we also construct a locally zeroth order coupling scheme (CE-0) by replacing (12) with

$$
f_{1}\left(x_{p d e}, t\right)=f_{1}^{[0]}\left(x_{p d e}, t\right)=\frac{1}{3} \rho\left(x_{p d e}, t\right)
$$

In both cases, we proceed afterwards with (13) and (14) as above.

For the above reconstruction schemes, the local error at the interface is defined as the dominant term left out in the Chapman-Enskog expansion. For example, the CE-1 scheme produces a second order interface error related to $f_{1}^{[2]}\left(x_{p d e}, t\right)(10)$ in each time step. In Sect. 5, we study the propagation of this error throughout the spatial domain.

\subsection{Implementation using Constrained Runs}

As stated in Sect. 3, the CR scheme produces a first order accurate approximation of the distribution functions. As an alternative to the procedure from Sect. 4.1, we can thus replace (12) with Algorithm 1 and apply (13) and (14) to the result.

Since information in the (explicit) LBE (4) propagates over only one lattice site in each iteration, Algorithm 1 requires initial density values on a sublattice with at least $2 K+1$ lattice sites, symmetrically distributed around $x_{p d e}$, where $K$ is the number of iterations needed for convergence of the algorithm. Arbitrary boundary conditions can be used on this sublattice because the boundary information will not have reached $x_{p d e}$ within $K$ iterations.

Alternatively, one can drop the outer lattice sites (and distribution functions) during propagation in each iteration to obtain a funneled scheme. Here we keep only the information streaming towards $x_{p d e}$. Again it is important that there are at least $2 K+1$ initial sites, symmetrically positioned around $x_{p d e}$. Note that this funneled scheme decreases the amount of work with a factor two. On the other hand, this implementation requires changes to the propagation step of the LBM in Algorithm 1, which may not be desirable.

The number of constrained runs $K$ can be obtained a-priori by a preliminary simulation on (part of) the domain with Algorithm 1 to observe its convergence (see also [36]). The amount of work needed by the CR scheme is on the order of $K^{2}$ lattice site updates. This can be an expensive overhead since the scheme has to be used in between each time step $\Delta t$. Of course, for situations where the analytical slaving relations (12) are unknown or difficult to obtain analytically, it is the only alternative. 


\section{Error Analysis at Steady State}

In this section, we derive closed expressions for the global spatial discretization error $E(x)$ of the hybrid steady state solution $\rho(x)$ defined by

$$
\rho(x)=\rho^{c}(x)+E(x),
$$

with $\rho^{c}(x)$ the exact steady state solution of (1). In Sect. 5.1 and Sect. 5.2 we study CE-0 respectively $\mathrm{CE}-1$ coupling. As the coupling with $\mathrm{CR}$ is also first order correct at the interface, the expressions in Sect. 5.2 will hold approximately. We use an appropriate example for each section. For CE-0 coupling, we consider pure diffusion. For CE-1 coupling, we use a reaction-diffusion system with constant reaction term. Both systems are provided with Dirichlet boundary conditions. Since the exact steady state solution of these model problems has zero second (respectively third) and higher order spatial derivatives, we expect that the global error is only related to the first (respectively second) derivative. The local error made in (16) respectively (12) concerns the same order of spatial derivatives. We discuss the practical use and generalization of these error expressions in Sect. 5.3. Finally, in Sect. 5.4, we study how the CE-1 results can be extended to a non-linear system.

\subsection{Error using CE-0 Coupling for Pure Diffusion}

Consider the purely diffusive system defined by (1) with $F(\rho(x, t))=0$ and Dirichlet boundary conditions. The evolution of density over one time step in the point $x_{l b e}$ closest to the interface in the LBM domain (see Fig. 1) is obtained by summing (4) over all distributions

$$
\begin{aligned}
\rho\left(x_{l b e}, t+\Delta t\right)= & (1-\omega)\left(f_{1}\left(x_{p d e}, t\right)+f_{0}\left(x_{l b e}, t\right)+f_{-1}\left(x_{l b e}+\Delta x, t\right)\right) \\
& +\frac{\omega}{3}\left(\rho\left(x_{p d e}, t\right)+\rho\left(x_{l b e}, t\right)+\rho\left(x_{l b e}+\Delta x, t\right)\right) .
\end{aligned}
$$

Here, $f_{1}\left(x_{p d e}, t\right)$ has the value $(16)$.

The steady state solution $\rho^{c}(x)$ of $(1)$ is a straight line connecting the boundary values. Second and higher order spatial derivatives of the solution (both exact and hybrid) are zero. As a consequence, the distributions on the LBM domain can be written as functionals up to first order in $\rho(8)-(9)$. Substitution in (18) leads to

$\rho\left(x_{l b e}, t+\Delta t\right)=\frac{1}{3}\left(\rho\left(x_{p d e}, t\right)+\rho\left(x_{l b e}, t\right)+\rho\left(x_{l b e}+\Delta x, t\right)\right)+(1-\omega) \frac{\Delta x}{3 \omega} \frac{\partial \rho\left(x_{l b e}+\Delta x, t\right)}{\partial x}$.

At steady state, we have $\partial \rho(x, t) / \partial t=0$ and $\rho\left(x_{l b e}, t+\Delta t\right)=\rho\left(x_{l b e}, t\right)$. Equation (19) reduces to

$$
0=\rho\left(x_{p d e}\right)-2 \rho\left(x_{l b e}\right)+\rho\left(x_{l b e}+\Delta x\right)+(1-\omega) \frac{\Delta x}{\omega} \frac{\mathrm{d} \rho\left(x_{l b e}+\Delta x\right)}{\mathrm{d} x} .
$$

For this model problem, (2) holds exactly for (discretized) $\rho^{c}(x)$ because the FD spatial discretization error is zero (see Sect. 2.1). We subtract (2) at steady state with $F\left(\rho^{c}(x)\right)=0$ from (20) to obtain

$$
0=E\left(x_{p d e}\right)-2 E\left(x_{l b e}\right)+E\left(x_{l b e}+\Delta x\right)+(1-\omega) \frac{\Delta x}{\omega} \frac{\mathrm{d} \rho\left(x_{l b e}+\Delta x\right)}{\mathrm{d} x} .
$$

To obtain derivatives of the exact instead of the hybrid solution, we substitute (17) into (21) and get

$$
\begin{aligned}
0= & 2 \omega E\left(x_{p d e}\right)-(1+3 \omega) E\left(x_{l b e}\right)+2 \omega E\left(x_{l b e}+\Delta x\right) \\
& +(1-\omega) E\left(x_{l b e}+2 \Delta x\right)+(1-\omega) 2 \Delta x \frac{\mathrm{d} \rho^{c}\left(x_{l b e}+\Delta x\right)}{\mathrm{d} x},
\end{aligned}
$$


where we discretized $\mathrm{d} E\left(x_{l b e}+\Delta x\right) / \mathrm{d} x$ with central differences around $x_{l b e}+\Delta x$.

Away from the interface inside the LBM domain, i.e. away from the instantaneous local error source at $x_{p d e}$ induced by (16), the incoming $f_{1}(x-\Delta x)$ is described by (8)-(9). Using the same reasoning that brought us from (18) to (20), we obtain the following equation for $\rho(x)$

$$
0=\rho(x-\Delta x)-2 \rho(x)+\rho(x+\Delta x)+(1-\omega) \frac{\Delta x}{\omega}\left(\frac{\mathrm{d} \rho(x+\Delta x)}{\mathrm{d} x}-\frac{\mathrm{d} \rho(x-\Delta x)}{\mathrm{d} x}\right) .
$$

Since the exact $\rho^{c}(x)$ is analytic on the whole domain and since $E(x)$ is analytic away from $x_{l b e}$, we use central differences for their derivatives on the LBM domain as follows

$$
\frac{\mathrm{d}\left\{\rho^{c}, E\right\}(x+\Delta x)}{\mathrm{d} x}-\frac{\mathrm{d}\left\{\rho^{c}, E\right\}(x-\Delta x)}{\mathrm{d} x}=2 \Delta x \frac{\mathrm{d}^{2}\left\{\rho^{c}, E\right\}(x)}{\mathrm{d} x^{2}} .
$$

Subtracting (2) at steady state from (23) and substituting (17) and (24), we obtain

$$
0=E(x-\Delta x)-2 E(x)+E(x+\Delta x)+(1-\omega) \frac{2 \Delta x^{2}}{\omega} \frac{\mathrm{d}^{2} E(x)}{\mathrm{d} x^{2}},
$$

where the term proportional to $\mathrm{d}^{2} \rho^{c}(x) / \mathrm{d} x^{2}=0$ cancels because (1) holds for $\rho^{c}$. From (25) it follows that the global error on the LBM domain is a solution of

$$
0=E(x-\Delta x)-2 E(x)+E(x+\Delta x),
$$

which means the discrete error points lie on a straight line. Using (2) and (17), it can be checked easily that the same holds on the PDE domain. Call $s_{1}$ and $s_{2}$ the slopes of the lines on the PDE respectively LBM domain. We then have

$$
E\left(x_{p d e}\right)=E\left(x_{l b e}\right)-s_{1} \Delta x, \quad E\left(x_{l b e}+\Delta x\right)=E\left(x_{l b e}\right)+s_{2} \Delta x .
$$

Since (2) is used up to $x_{p d e}$, the top of this tent-like function lies at $x_{l b e}$. Furthermore, with Dirichlet boundary conditions, we have $E(0)=0$ and $E(L)=0$. The slopes $s_{1}$ and $s_{2}$ are then related by $s_{1} L_{1}=-s_{2} L_{2}$ with $L_{1}=x_{l b e}$ and $L_{2}=L-L_{1}$. By substituting (27) into (22), an expression for $s_{1}$ can be found.

Putting all of this together, we can conclude that the maximal error is attained at $L_{1}=x_{l b e}$ and has the value $E_{0}=E\left(x_{l b e}\right)=s_{1} L_{1}$, i.e.

$$
E_{0}=\frac{L_{1} L_{2}}{L_{1}+L_{2} \omega}(1-\omega) \frac{\mathrm{d} \rho^{c}\left(x_{l b e}+\Delta x\right)}{\mathrm{d} x} .
$$

\subsection{Error using CE-1 Coupling for Constant Reaction Term}

Here, we consider a reaction-diffusion system (1) with constant reaction term and Dirichlet boundary conditions. The density in $x_{l b e}$ evolves in each time step as

$$
\begin{aligned}
\rho\left(x_{l b e}, t+\Delta t\right) & =(1-\omega)\left(f_{1}\left(x_{\text {pde }}, t\right)+f_{0}\left(x_{l b e}, t\right)+f_{-1}\left(x_{l b e}+\Delta x, t\right)\right) \\
& +\frac{\omega}{3}\left(\rho\left(x_{p d e}, t\right)+\rho\left(x_{l b e}, t\right)+\rho\left(x_{l b e}+\Delta x, t\right)\right)+\Delta t F\left(\rho\left(x_{l b e}, t\right)\right) \\
& +\frac{\Delta t}{3}\left(F\left(\rho\left(x_{p d e}, t\right)\right)-2 F\left(\rho\left(x_{l b e}, t\right)\right)+F\left(\rho\left(x_{l b e}+\Delta x, t\right)\right)\right)
\end{aligned}
$$

where $f_{1}\left(x_{p d e}, t\right)$ is computed with (12). The last line in (29) equates to zero when $F(\rho)$ is constant and can be dropped.

The exact steady state solution $\rho^{c}(x)$ now has a parabolic shape. Third and higher order spatial derivatives of the solution are zero. The distributions on the LBM domain can thus be written as functionals up to second order in $\rho(8)-(10)$. It 
can be checked that substitution of (12) for $f_{1}\left(x_{p d e}\right)$ and (8)-(10) for $f_{0}\left(x_{l b e}\right)$ and $f_{-1}\left(x_{l b e}+\Delta x\right)$ in $(29)$ at steady state results in

$$
\begin{aligned}
0= & \frac{1}{3}\left(\rho\left(x_{p d e}\right)-2 \rho\left(x_{l b e}\right)+\rho\left(x_{l b e}+\Delta x\right)\right)+(1-\omega) \frac{\Delta x}{3 \omega}\left(\frac{\mathrm{d} \rho\left(x_{l b e}+\Delta x\right)}{\mathrm{d} x}-\frac{\mathrm{d} \rho\left(x_{p d e}\right)}{\mathrm{d} x}\right) \\
& +\Delta t F\left(\rho\left(x_{l b e}\right)\right)+(1-\omega) \frac{\Delta x^{2}}{18 \omega^{2}}(\omega-2)\left(2 \frac{\mathrm{d}_{+}^{2} \rho\left(x_{l b e}\right)}{\mathrm{d} x^{2}}-\frac{\mathrm{d}^{2} \rho\left(x_{l b e}+\Delta x\right)}{\mathrm{d} x^{2}}\right) .
\end{aligned}
$$

Note that $\mathrm{d}_{+}^{2} \rho\left(x_{l b e}\right) / \mathrm{d} x^{2}$ is defined on the LBM domain only (the hybrid $\rho(x)$ is nondifferentiable at $x_{l b e}$ and right hand differences should be used when discretizing).

As in Sect. 5.1, the FD spatial discretization error of (2) is zero because all higher-than-second derivatives are zero. Consequently, $(2)$ holds exactly for $\rho^{c}(x)$. Multiplying this expression by $1 / 3$ and subtracting the result from (30), we obtain the following for the error at steady state

$$
\begin{aligned}
0= & \frac{1}{3}\left(E\left(x_{p d e}\right)-2 E\left(x_{l b e}\right)+E\left(x_{l b e}+\Delta x\right)\right)+\Delta t F\left(\rho\left(x_{l b e}\right)\right) \\
& -\frac{\Delta x^{2}}{3 D} F\left(\rho^{c}\left(x_{l b e}\right)\right)+(1-\omega) \frac{\Delta x}{3 \omega}\left(\frac{\mathrm{d} \rho\left(x_{l b e}+\Delta x\right)}{\mathrm{d} x}-\frac{\mathrm{d} \rho\left(x_{p d e}\right)}{\mathrm{d} x}\right) \\
& +(1-\omega) \frac{\Delta x^{2}}{18 \omega^{2}}(\omega-2)\left(2 \frac{\mathrm{d}_{+}^{2} \rho\left(x_{l b e}\right)}{\mathrm{d} x^{2}}-\frac{\mathrm{d}^{2} \rho\left(x_{l b e}+\Delta x\right)}{\mathrm{d} x^{2}}\right) .
\end{aligned}
$$

We now substitute (17) into (31). Since $\rho^{c}(x)$ is analytic on the whole domain, we can use (24) around $x_{l b e}$ for its derivatives. As the reaction terms are independent of $\rho$ for our model problem, they both cancel with (24) in (31) because (1) holds for $\rho^{c}$. By contrast, we can not use (24) around $x_{l b e}$ for the derivatives of $E(x)$ because we expect $E(x)$ to be non-differentiable at $x_{l b e}$. Instead, we discretize $\mathrm{d} E\left(x_{l b e}+\Delta x\right) / \mathrm{d} x$ and $\mathrm{d} E\left(x_{p d e}\right) / \mathrm{d} x$ separately with central differences around $x_{l b e}+\Delta x$ and $x_{p d e}$. We then obtain a relation in terms of derivatives of the exact solution

$$
\begin{aligned}
0 & =(1-\omega) E\left(x_{p d e}-\Delta x\right)+2 \omega E\left(x_{p d e}\right)-2(1+\omega) E\left(x_{l b e}\right)+2 \omega E\left(x_{l b e}+\Delta x\right) \\
& +(1-\omega) E\left(x_{l b e}+2 \Delta x\right)+(1-\omega) \frac{\Delta x^{2}}{3 \omega}(\omega-2)\left(2 \frac{\mathrm{d}^{2} \rho^{c}\left(x_{l b e}\right)}{\mathrm{d} x^{2}}-\frac{\mathrm{d}^{2} \rho^{c}\left(x_{l b e}+\Delta x\right)}{\mathrm{d} x^{2}}\right) \\
& +(1-\omega) \frac{\Delta x^{2}}{3 \omega}(\omega-2)\left(2 \frac{\mathrm{d}_{+}^{2} E\left(x_{l b e}\right)}{\mathrm{d} x^{2}}-\frac{\mathrm{d}^{2} E\left(x_{l b e}+\Delta x\right)}{\mathrm{d} x^{2}}\right) .
\end{aligned}
$$

Away from the interface, there is no instantaneous local error contribution (12) and the incoming $f_{1}(x-\Delta x)$ can be written as (8)-(10). Inside the LBM domain, we have an equation for $\rho(x)$ very similar to $(30)$, except that it has a contribution

$$
-(1-\omega) \frac{\Delta x^{2}}{18 \omega^{2}}(\omega-2)\left(\frac{\mathrm{d}^{2} \rho(x-\Delta x)}{\mathrm{d} x^{2}}-2 \frac{\mathrm{d}^{2} \rho(x)}{\mathrm{d} x^{2}}+\frac{\mathrm{d}^{2} \rho(x+\Delta x)}{\mathrm{d} x^{2}}\right),
$$

instead of just the last two terms in (30). In (33), the terms between brackets can be replaced by the shorthand $\Delta x^{2} \mathrm{~d}^{4} \rho(x) / \mathrm{d} x^{4}$. When substituting (17) into (33), we get a term proportional to $\mathrm{d}^{4} \rho^{c}(x) / \mathrm{d} x^{4}=0$ and one proportional to $\mathrm{d}^{4} E(x) / \mathrm{d} x^{4}$. The latter is explicitly presumed to be negligible here. Skipping a few steps elaborated on previously, we find that, except for $x_{l b e}$, the global error $E(x)$ on both the LBM and PDE domain is a solution of (26). Again, we obtain a tent-shaped error function with top at $x_{l b e}$ and the relation $s_{1} L_{1}=-s_{2} L_{2}$ between the slopes $s_{1}$ and $s_{2}$. By substituting (27) into (32), an expression for $s_{1}$ can be found. Because $E(x)$ is 
piecewise linear, its second derivatives are zero and the last term in (32) cancels. The maximal error is attained at $L_{1}=x_{l b e}$ and has the value $E_{1}=E\left(x_{l b e}\right)=s_{1} L_{1}$

$$
E_{1}=\frac{L_{1} L_{2}}{L}(1-\omega) \frac{\Delta x}{6 \omega}(\omega-2)\left(2 \frac{\mathrm{d}^{2} \rho^{c}\left(x_{l b e}\right)}{\mathrm{d} x^{2}}-\frac{\mathrm{d}^{2} \rho^{c}\left(x_{l b e}+\Delta x\right)}{\mathrm{d} x^{2}}\right) .
$$

\subsection{Discussion}

When $\omega$ is kept constant, the global steady state error (28) of the CE-0 scheme remains constant as $\Delta x$ decreases; i.e. it is of zeroth order in $\Delta x$. This is opposed to the instantaneous local error made in each time step which is of first order (cf. Sect. 4.1). Similarly, the global error (34) of the CE-1 scheme is of first order in $\Delta x$ for constant $\omega$, while the local error is of second order. In this paper, we will not derive a closed expression for the error of the CE-2 coupling scheme, but we verify numerically in Fig. 5 (left) that using the CE-2 coupling scheme (with a third order local error) also leads to an accuracy loss of one order, i.e. we obtain a second order global error. We also show in Sect. 6 that the results with the CE-1 scheme are sufficiently accurate for practical computations.

By substituting (27) into (21) instead of (22) we can obtain an alternate expression for $E_{0}$ in terms of the derivative of the hybrid solution

$$
E_{0}=\frac{L_{1} L_{2}}{L}(1-\omega) \frac{1}{\omega} \frac{\mathrm{d} \rho\left(x_{l b e}+\Delta x\right)}{\mathrm{d} x} .
$$

For CE-1 coupling, the derivatives in (34) can be replaced by those of the hybrid density also because $E(x)$ is piecewise linear (compare (31) and (32)). Such expressions are more practical than (28) and (34) to compute the a-posteriori error of the hybrid system. As $\rho^{c}(x)=\rho(x)-E(x)$, the $E(x)$ values could even be used to correct the hybrid solution.

In the above accuracy analysis, we studied the propagation of a highly localized error in space and its influence on the global error on the full domain. We focused on LB/FD hybrid models, but the problem is more general. Loosely stated, we solve the following continuous point source equation for the global steady state error $E(x)$

$$
\frac{\mathrm{d}^{2} E(x)}{\mathrm{d} x^{2}}+\tilde{F}(E(x))=A \Delta x^{p} \delta\left(x-x_{l b e}\right),
$$

where $\tilde{F}(E(x))$ is a problem-specific force term; the power $p$ is determined by the coupling scheme and the Dirac delta function $\delta\left(x-x_{l b e}\right)$ represents the localized error source at the interface $x_{l b e}$. The amplitude $A$ is also problem-specific and contains derivatives of the solution around $x_{l b e}$. Equation (36) can be retrieved from the combination of (21) and (26) for CE-0 coupling and (32)-(26) for CE-1 coupling.

\subsection{Error using CE-1 Coupling for Non-Linear Reaction Term}

In this section, we study how the results from Sect. 5.2 extend to reaction-diffusion systems with a reaction term which is non-linear in $\rho$. The Fisher equation (1) with reaction term $F(\rho)=\alpha \rho(1-\rho), \alpha \in \mathbb{R}$ and Dirichlet boundary conditions serves as our model problem.

Again, we focus on the interface first. The evolution of $\rho\left(x_{l b e}, t\right)$ in one time step is still described by $(29)$ with $f_{1}\left(x_{p d e}, t\right)$ given by $(12)$. However, the last line in (29) can not be dropped.

Because of the non-linearity, higher order spatial derivatives of the solution will be non-zero. This leads to two main approximations. First, substitution of the Chapman-Enskog slaving relations (8)-(10) up to second order in (29) at steady 
state still leads to (30) (including the last line of (29)) but (30) is now only an approximation. Second, (2) will no longer hold exactly for $\rho^{c}(x)$ because of the now non-zero FD discretization error. Therefore, we redefine $E(x)$ as

$$
\rho(x)=\rho^{F D}(x)+E(x),
$$

with $\rho^{F D}(x)$ the FD solution of (2). We then subtract (2) from (30) to obtain equation (31) for $E(x)$ defined by (37).

We proceed further from (31) by substituting (37). For the Fisher equation, the dominant force terms in (31) result in a contribution

$$
\left(\Delta t-\frac{\Delta x^{2}}{3 D}\right) F\left(\rho^{F D}\left(x_{l b e}\right)\right)+\Delta t \alpha\left(1-2 \rho^{F D}\left(x_{l b e}\right)\right) E\left(x_{l b e}\right)-\Delta t \alpha E^{2}\left(x_{l b e}\right) .
$$

As in Sect. 5.2, the first term in (38) cancels with (24). For practical computations, (37) can be substituted in the remainder of (38) to obtain an expression which is independent of $\rho^{F D}\left(x_{l b e}\right)$. The approximate equation for $E\left(x_{l b e}\right)$ is then given by

$$
\begin{aligned}
0 & =(1-\omega) E\left(x_{p d e}-\Delta x\right)+2 \omega E\left(x_{p d e}\right)-2(1+\omega) E\left(x_{l b e}\right)+2 \omega E\left(x_{l b e}+\Delta x\right) \\
& +(1-\omega) E\left(x_{l b e}+2 \Delta x\right)+6 \omega \Delta t \alpha\left(1-2 \rho\left(x_{l b e}\right)\right) E\left(x_{l b e}\right)+6 \omega \Delta t \alpha E^{2}\left(x_{l b e}\right) \\
& +2 \omega \Delta t\left(F\left(\rho\left(x_{p d e}\right)\right)-2 F\left(\rho\left(x_{l b e}\right)\right)+F\left(\rho\left(x_{l b e}+\Delta x\right)\right)\right) \\
& +(1-\omega) \frac{\Delta x^{2}}{3 \omega}(\omega-2)\left(2 \frac{\mathrm{d}_{+}^{2} \rho\left(x_{l b e}\right)}{\mathrm{d} x^{2}}-\frac{\mathrm{d}^{2} \rho\left(x_{l b e}+\Delta x\right)}{\mathrm{d} x^{2}}\right),
\end{aligned}
$$

where we kept the last line in terms of hybrid derivatives for computational purpose (we want to compute the a-posteriori error).

Away from the interface inside the LBM domain, $E(x)$ is an approximate solution of (39) without the last line. Alternatively, we can use (24) for the first derivatives of $E(x)$ and find that $E(x)$ is the solution of

$$
\begin{aligned}
0= & (2-\omega)(E(x-\Delta x)-2 E(x)+E(x+\Delta x))+3 \omega \Delta t \alpha(1-2 \rho(x)) E(x) \\
& +3 \omega \Delta t \alpha E^{2}(x)+\omega \Delta t(F(\rho(x-\Delta x))-2 F(\rho(x))+F(\rho(x+\Delta x))) .
\end{aligned}
$$

On the PDE domain, the hybrid $\rho(x)$ and $\rho^{F D}(x)$ are approximate solutions of (2) at steady state (up to the FD discretization error). Subtracting these expressions from each other (and using relation (5) between $D$ and $\omega$ ), we obtain an equation similar to $(40)$

$$
0=\frac{2-\omega}{3 \omega}(E(x-\Delta x)-2 E(x)+E(x+\Delta x))+\Delta t \alpha(1-2 \rho(x)) E(x)+\Delta t \alpha E^{2}(x) .
$$

The system, consisting of (41) on the PDE subdomain, (39) at $x_{l b e}$ and (40) on the remainder of the LBM domain, can be solved for $E(x)$ with boundary conditions $E(0)=E(L)=0$. As a further approximation, the non-linear term $\Delta t \alpha E^{2}(x)$ can be dropped. Otherwise, Newton's method can be used. In our tests for Example 6.3 , the latter had a negligible impact on the result.

\section{Numerical Results}

In this section, we construct the hybrid LB/FD models from Sect. 4 for several reaction-diffusion examples with appropriate Dirichlet boundary conditions. For the LBM, the boundary condition is implemented as in [36]. We also compute the spatial discretization error to illustrate the theoretical results from Sect. 5. 

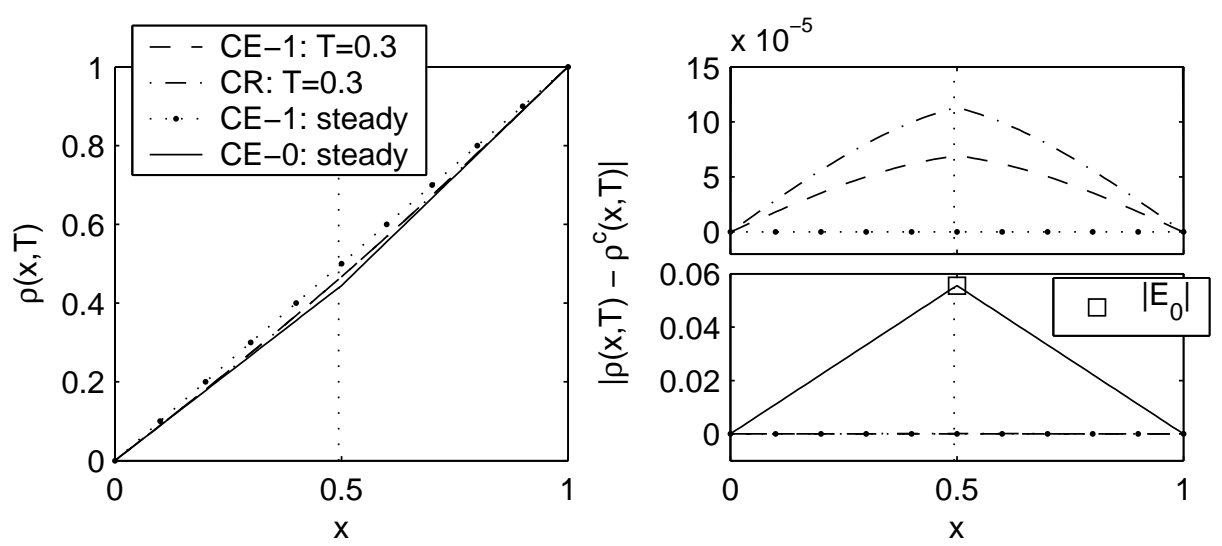

Figure 2: Hybrid density (left) and corresponding error (right) with respect to the exact solution at time $t=T=0.3$ and at steady state for Example 6.1. Results for CE-0, CE-1 and CR coupling are shown. The square marks the theoretical $\left|E_{0}\right|$ value (28). Notice the scale difference in the right figures. The dotted line denotes the interface.

Example 6.1 (Pure diffusion) Consider the purely diffusive problem (1) with $F(\rho(x, t))=0$ on the one-dimensional domain $[0, L]$ with Dirichlet boundary conditions $\rho(0, t)=0, \rho(L, t)=1$ and initial condition $\rho(x, 0)=0$. The exact timedependent solution can be computed as

$$
\rho^{c}(x, t)=\sum_{n=1}^{\infty}(-1)^{n} \frac{2}{n \pi} \exp \left(-\frac{D n^{2} \pi^{2} t}{L^{2}}\right) \sin \left(\frac{n \pi x}{L}\right)+\frac{x}{L} .
$$

The steady state solution equals $\rho^{c}(x)=x / L$. The same example was used in [2, 3].

In Fig. 2, we plot the hybrid density $\rho(x, T)$ (left) and the corresponding error $\left|\rho(x, T)-\rho^{c}(x, T)\right|$ in absolute value (right) for Example 6.1. Results are shown for $t=T=0.3$ and for $t=T=10$, where the latter approximates the steady state. Parameters are $D=1, L=1, L_{1}=0.5$ and $K=10$. The number of lattice points $N=81(\Delta x=1 / 80)$ while $\Delta t=3.125 \mathrm{e}-5$ such that $\omega=1.25(5)$. As the steady state in a purely diffusive problem only has a non-zero first derivative, the errors of CE-1 and CR coupling die out as time increases (the latter because (11) is proven first order accurate). The fact that CR coupling is a little less accurate than CE-1 coupling can be attributed to the crude approximation of the time derivative in the local error made in (11) compared to (10).

Observe that coupling with the CE-0 scheme (16) results in a tent-shaped error at steady state as predicted in Sect. 5.1. This steady state behavior can also be observed in $[2,3]$. Its maximal value is attained at $x_{l b e}=L_{1}$ and can be computed as $\max \left|\rho(x)-\rho^{c}(x)\right|=0.0555$, which corresponds up to 13 digits to the theoretical value $\left|E_{0}\right|$ from (28) and (35). It can be checked that this error remains constant as $\Delta x$ is decreased for constant $\omega$.

Next, we compute the discretization error of the CE-1 coupling scheme for Example 6.1. For an increasing number of discretization points $N=21,41,81,161$, 321 , i.e. for decreasing values of $\Delta x$, we compute the hybrid solution at time $T=0.3$ (away from the steady state) and compare to the exact solution (42).

Because $\Delta x$ and $\Delta t$ are interdependent through $\omega(5)$, we alter $\Delta t$ when decreasing $\Delta x$ along a refinement path of constant $\omega$. We kept $\Delta t / \Delta x^{2}=1 / 5$ such that $\omega=1.25$. This way, the hybrid solution relaxes in the same way for each refinement. This is different from the approach taken in [3]. There, the authors fix 

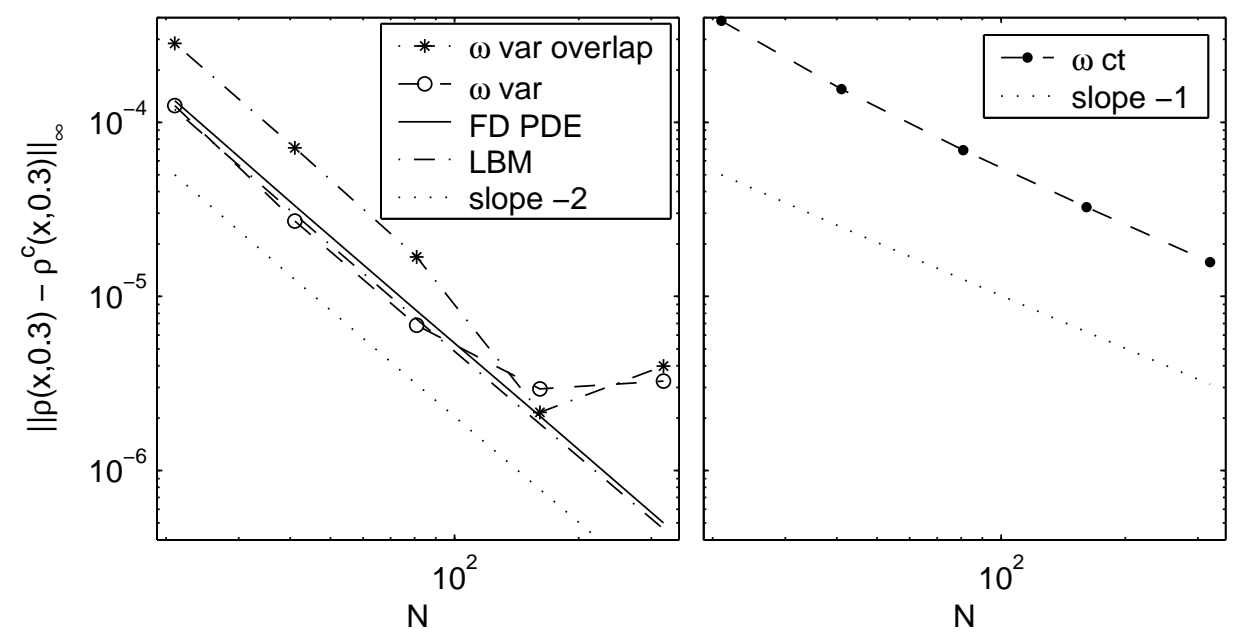

Figure 3: Log-log plot of the discretization error at time $T=0.3$ as a function of $N$ for the hybrid model from Example 6.1 with CE-1 coupling. The hybrid coupling scheme for our setting (constant $\omega$ ) is only first order accurate in space (right). Results for variable $\omega$, both for the implementation with one overlapping site from Albuquerque et al. $[2,3]$ (labeled $\omega$ var overlap) and our implementation from Sect. 4 (labeled $\omega$ var), are shown to the left, together with the second order behavior of the FD PDE and LBM on the full domain. Dotted lines with slopes -1 and -2 are added for convenience.

a very small value of $\Delta t$ by adjusting the value of $\omega$. In our opinion, this is done to keep only the error contribution in terms of $\Delta x$ dominant. Note that for small $\Delta t$, $\omega$ approximates 2 , which is not a very interesting simulation regime.

Figure 3 (right) shows the maximum (infinity) norm of the discretization error at $T=0.3$ using $\mathrm{CE}-1$ coupling for our setting (constant $\omega$ ). Although we have a second order error locally in (12), we observe only first order behavior globally as predicted by (34). To the left, the error obtained with varying $\omega$ (and $\Delta t=1 \mathrm{e}-7$ ) is shown, both for our implementation and for the implementation from $[2,3]$ (using one overlapping site and also first order slaving relations (9)). The discretization error appears to be second order for the first few refinements, as reported in $[2,3]$, but for smaller $\Delta x$ - the case of interest when looking at discretization errors - this is clearly no longer the case. In Fig. 5 we will come back to this. For completeness, we also show the (second order) error of both the LBM (with constant $\omega$ ) and PDE simulated independently on the full domain. As a guide to the eye, dotted lines with slope -2 (left) and -1 (right) are drawn. We observe in the left figure that the use of an overlapping site hardly changes the results. Note that the result with an overlapping site and constant $\omega$ is also first order accurate (not shown).

As the steady state for pure diffusion only has a non-zero first derivative, we now turn our attention to an example with a non-zero second derivative to study the steady state error of the CE-1 coupling scheme.

Example 6.2 (Constant reaction term) Consider a reaction-diffusion system (1) with constant reaction term $F(\rho(x, t))=2 D$. We impose Dirichlet boundary conditions $\rho(0, t)=\rho(L, t)=0$ and initial condition $\rho(x, 0)=0$. The exact solution can be derived as

$\rho^{c}(x, t)=\sum_{n=0}^{\infty} \frac{-8 L^{2}}{(2 n+1)^{3} \pi^{3}} \exp \left(-\frac{D(2 n+1)^{2} \pi^{2} t}{L^{2}}\right) \sin \left(\frac{(2 n+1) \pi x}{L}\right)+x(L-x)$. 

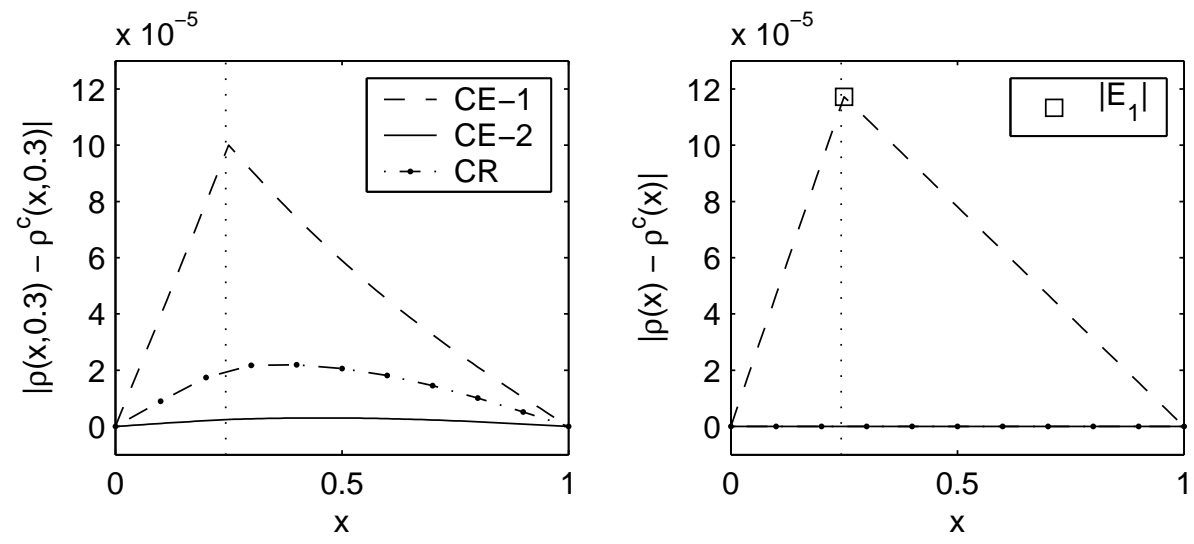

Figure 4: Error of the hybrid density w.r.t. the exact solution (43) at time $t=T=0.3$ (left) and at steady state (right) for Example 6.2. The square marks the theoretical $\left|E_{1}\right|$ value (34). The dotted line denotes the interface.

The steady state solution is $\rho^{c}(x)=x(L-x)$ with second spatial derivative -2 .

Figure 4 shows the error of the hybrid solution from Example 6.2 at time $t=$ $T=0.3$ (left) and at steady state $(T=10)$ (right). Parameters are $D=1, L=1$, $L_{1}=0.25, K=13, \Delta x=1 / 80$ and $\Delta t=3.125 \mathrm{e}-5$. In addition to the local coupling with CE-1 and CR, we also implemented the second order CE-2 coupling scheme (15). A few observations can be made. First, the steady state error for CE-1 coupling behaves as predicted in Sect. 5.2, with $\left|E_{1}\right|=1.172 \mathrm{e}-4$. Second, CR coupling is more accurate than CE-1 coupling for this example (compare to Fig. 2, where we observed the opposite). This can be explained by comparing (11) to (9)-(10). We see that the CR scheme correctly computes the force term in the second order contribution $f_{1}^{[2]}\left(x_{p d e}, t\right)$, which CE-1 coupling obviously does not do. Finally, because the steady state solution for this model problem has non-zero spatial derivatives up to second order only and $\partial \rho / \partial t=0$ in $(10)$ and $\tilde{\rho}-\rho^{(0)}=0$ in (11), the error for both the CE-2 as well as the CR coupling scheme approaches zero as time increases.

In Fig. 5, we plot the infinity norm of the discretization error using CE-1 coupling as a function of the number of discretization points $N$. Again, we choose $\Delta t$ such that $\omega=1.25$ for all $\Delta x$. Shown left is the steady state discretization error (at $T=10$ ) for our setting compared to the expression for the maximum defined by (34). These values correspond up to at least 13 digits. The global error behavior is again first order in space. Figure 5 also shows numerically that the global discretization error for CE-2 coupling is of second order when $\omega$ is kept constant, which confirms our statement in Sect. 5.3. It follows that the global error of the CR scheme at steady state is second order also.

The right panel of Fig. 5 shows the discretization error of the CE-1 hybrid model with variable $\omega$ and constant but small $\Delta t=1 \mathrm{e}-7$ (for our implementation without overlap). As the simulation time is increased from $T=0.04$ over $T=0.3$ and 0.6 to $T=10$ (the steady state), we see that the apparent second order behavior breaks down and is merely an artefact of the choice for a constant $\Delta t$. At steady state the error is proportional to $N=1 / \Delta x$. In this case also, the theoretical $E_{1}$ value (34) is in excellent accordance with the numerical steady state error.

Example 6.3 (Non-linear system) Consider the Fisher equation defined by (1) with $F(\rho(x, t))=50 \rho(x, t)(1-\rho(x, t))$. We apply Dirichlet boundary conditions 

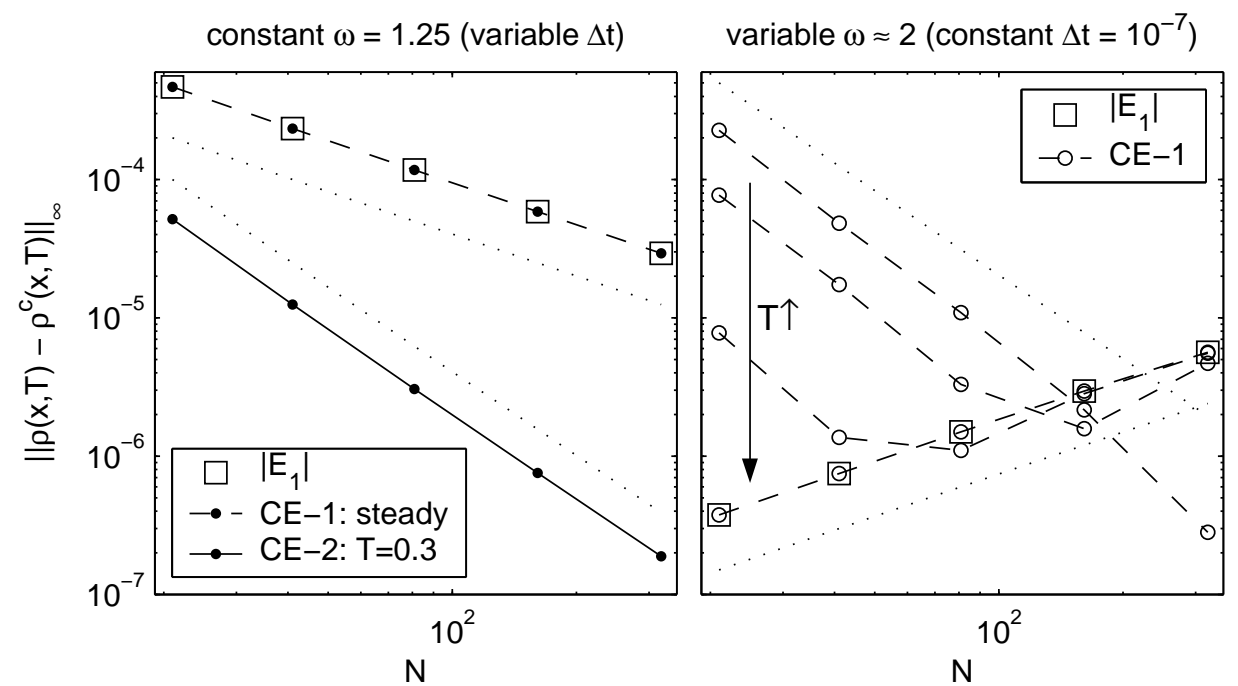

Figure 5: Log-log plot of the discretization error of the hybrid model with CE-1 coupling for Example 6.2 w.r.t. $N$. Left: the steady state error computed with our CE-1 hybrid model as well as the error at $T=0.3$ for CE- 2 coupling. Right: The error for the CE-1 hybrid model with variable $\omega$ (and no overlap) at respective times $T=0.04,0.3,0.6$ and 10 (the latter at steady state). It is clear that the initially apparent second order is an artefact. In both figures, expression (34) for the maximal CE-1 error is also plotted. Dotted lines with slopes $-1,-2$ (left) and $-2,+1$ (right) are added as a guide to the eye.

$\rho(0, t)=\rho(L, t)=0$ and use $\rho(x, 0)=x(L-x)$ as initial condition. The exact steady state solution is approximated by the PDE (or the LBM) solution on the full domain for fine $\Delta x=1 / 2560$.

We choose $L_{1}=0.6$ and use the same values as in the previous two examples for the other system parameters. In Fig. 6, we plot the steady state error $E(x)$ of the CE-1 hybrid model for Example 6.3, computed numerically from the equations described in Sect. 5.4. To the left, we compare $E(x)$ to the density difference of a) the hybrid and "exact" solution and b) the hybrid and PDE solution on the full domain for $\Delta x=1 / 80$. To the right, we compare $E(x)$ to a measure for the modeling error, being the difference between the solution of a LBM and PDE simulation on the full domain for $\Delta x=1 / 80$.

While the hybrid error is again non-differentiable at $x_{l b e}=L_{1}$, its maximum is now clearly attained elsewhere due to the non-linearity of the system. Because we had to redefine $E(x)$ to $(37)$ in Sect. 5.4, the $E(x)$ curve is a better approximation of the error w.r.t. the FD solution for $\Delta x=1 / 80$ than w.r.t. the exact solution. If we compare $E(x)$ to the modeling error between LBM and PDE (Fig. 6 right), we see that the accuracy of CE-1 coupling is sufficient for practical computations.

Finally, we plot $\left\|\rho(x)-\rho^{c}(x)\right\|_{\infty}$ for the CE-1 hybrid model at $T=10$ (the steady state) in Fig. 7. On the bottom axis, we increased the spatial resolution to $N=1281$. We compare these values to the measure for the modeling error defined before and to $\left|\rho\left(x_{l b e}\right)-\rho^{c}\left(x_{l b e}\right)\right|$. Clearly, the second order modeling error is the dominant contribution to the error for values of $\Delta x$ up to $1 / 160$. The maxima of the hybrid and modeling error agree reasonably well and are attained at the same positions (see Fig. 6). For values of $\Delta x=1 / 320$ and smaller (i.e. $N>161$ ), the first order contribution (due to the spatial coupling) in the global error becomes predominant and its maximum is attained at the interface $x_{l b e}$ as Fig. 7 indicates. 


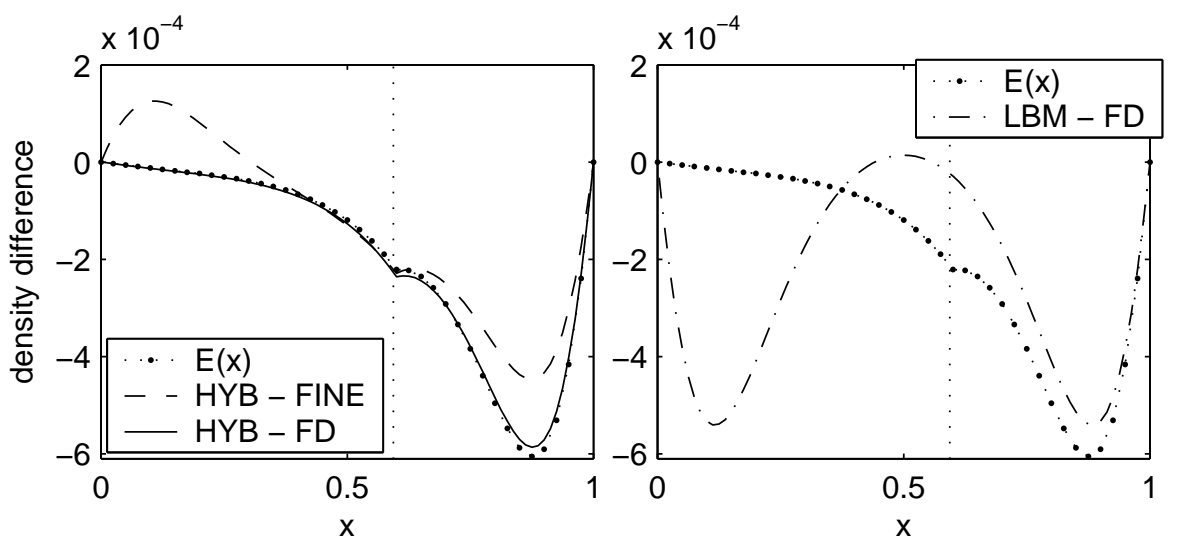

Figure 6: Steady state error $E(x)$ of the CE-1 hybrid model for the Fisher Example 6.3 computed as described in Sect. 5.4. Also shown to the left: the difference $\rho(x)-\rho^{c}(x)$, with $\rho^{c}(x)$ approximated by the FD PDE solution on a very fine lattice (labeled HYB FINE) and the difference of the hybrid w.r.t. the FD density for $\Delta x=1 / 80$ (labeled HYB - FD). To the right, $E(x)$ is compared to the modeling error, i.e. the density difference between the LBM and FD PDE on the full domain for $\Delta x=1 / 80$ (labeled LBM - FD). The dotted line denotes the interface.

More numerical results for a growth-diffusion problem and a reaction-diffusion problem with two interacting species can be found in [37].

\section{Conclusions}

In this paper, we studied the hybrid models obtained by spatially coupling a lattice Boltzmann model (LBM) and a finite difference discretization (FD) of a partial differential equation (PDE), each defined on separate regions of the spatial domain. We focused on the analysis of the spatial discretization error of the hybrid models for reaction-diffusion systems on a one-dimensional domain.

At the interface between the two models, we have to map the PDE density to more LBM distribution functions. This mapping problem can be solved analytically with relations derived from the Chapman-Enskog expansion that express the distribution functions as a functional of the density and its derivatives - an idea first introduced in $[2,3]$ — or numerically with the iterative constrained runs scheme $[19,18]$ that approximates these relations numerically up to first order (and up to second order at steady state) [36]. The second method is useful when the ChapmanEnskog based relations are unavailable or difficult to obtain analytically, but is computationally much more expensive. At the interface, we used the Chapman-Enskog relations up to zeroth (labeled $\mathrm{CE}-0)$, first ( $\mathrm{CE}-1)$ and second (CE-2) order. The local error at the interface is then defined as the dominant term left out in this expansion.

Most importantly, we have shown that the global spatial discretization error of the hybrid model is one order less accurate than the local error made at the interface. We derived closed expressions for the global steady state discretization error of the hybrid model for the CE-0 and CE-1 coupling scheme. Furthermore, we have shown how these results can be extended when the reaction term is non-linear by deriving approximate equations from which the steady state error can be computed. The results were verified numerically for several model problems. We also showed that the accuracy of the CE- 1 scheme is sufficient for most practical computations by 


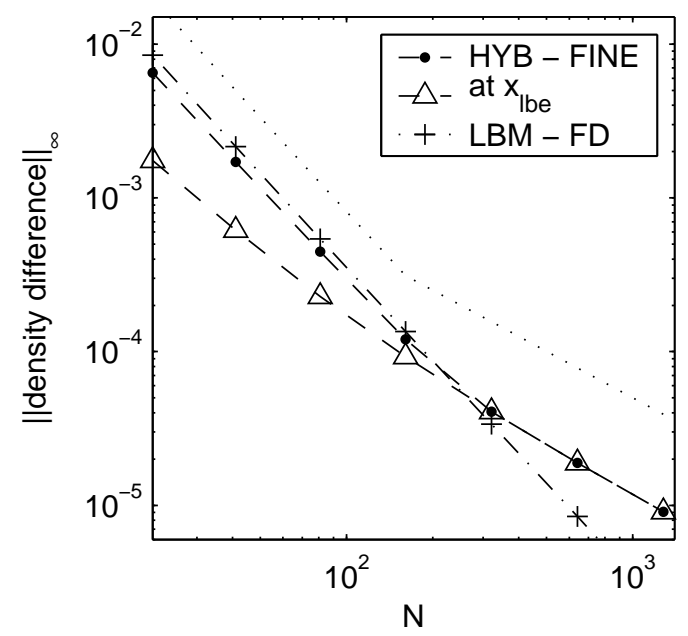

Figure 7: Log-log plot of the steady state error of the hybrid model with CE-1 coupling for Example 6.3 w.r.t. $N$. The labels denote the same as in Fig. 6 . In addition, the values $\left|\rho\left(x_{l b e}\right)-\rho^{c}\left(x_{l b e}\right)\right|$ are plotted. The dotted lines with slopes -2 and -1 guide the eye.

factoring in the modeling error between the LBM and PDE. On the other hand, only the use of the CE-2 scheme assures a second order error behavior globally.

For analysis purposes, we used the same space and time step $\Delta x$ and $\Delta t$ on both sublattices. The focus was on the details of the coupling and how information is exchanged. The use of different $\Delta x$ and $\Delta t$ values in both regions optimized to local stability properties would be a further development, see $[5,9,15,16,23,26$, $31,33,38]$ and $[6,7,27,32,34,39]$ for guidelines. Also, we used LBM and PDE models which are equivalent in the macroscopic limit. However, we expect that the results can be carried over to LB/FD hybrid models where the submodels describe distinct dynamical behavior. It is important that the interface is situated in an intermediate region where the evolution is described well by both the LBM and PDE such that the relations from the Chapman-Enskog expansion are valid there.

\section{Acknowledgements}

The research in this paper is funded by projects G.0130.03 and G.0365.06 (PVL) of the Fund for Scientific Research - Flanders and by project OT/03/04 (CV) of the Research Fund of the K.U.Leuven. The paper presents research results of the Belgian Programme on Interuniversity Attraction Poles, initiated by the Belgian Federal Science Policy Office, who also supported WV with a DWTC return grant.

\section{References}

[1] P. Ahlrichs and B. Dünweg. Simulation of a single polymer chain in solution by combining lattice Boltzmann and molecular dynamics. Journal of Chemical Physics, 111(17):8225-8239, 1999.

[2] P. Albuquerque, D. Alemani, B. Chopard, and P. Leone. Coupling a lattice Boltzmann and a finite difference scheme. In M. Bubak, G. D. van Albada, P. M. Sloot, and J. Dongarra, editors, International Conference on Computational Science - ICCS 2004, volume 3039 of Lecture Notes in Computer Science, pages 540-547. SpringerVerlag, 2004. 
[3] P. Albuquerque, D. Alemani, B. Chopard, and P. Leone. A hybrid lattice Boltzmann finite difference scheme for the diffusion equation. International Journal for Multiscale Computational Engineering, 4(2):209-219, 2006.

[4] F. J. Alexander, A. L. Garcia, and D. M. Tartakovsky. Algorithm refinement for stochastic partial differential equations: I. Linear diffusion. Journal of Computational Physics, 182:47-66, 2002.

[5] M. J. Berger and J. Oliger. Adaptive mesh refinement for hyperbolic partial differential equations. Journal of Computational Physics, 53(3):484-512, 1984.

[6] M. Bernaschi, S. Succi, and H. Chen. Accelerated lattice Boltzmann schemes for steady-state flow simulations. Journal of Scientific Computing, 16(2):135-144, 2001.

[7] M. Bernaschi, S. Succi, H. Chen, and R. Zhang. Computing steady state flows with an accelerated lattice Boltzmann technique. International Journal of Modern Physics $C, 13(5): 675-687,2002$.

[8] A. Caiazzo. Analysis of lattice Boltzmann initialization routines. Journal of Statistical Physics, 121(1-2):37-48, 2005.

[9] N. Cao, S. Chen, S. Jin, and D. Martínez. Physical symmetry and lattice symmetry in the lattice Boltzmann method. Physical Review E, 55(1):R21-R24, 1997.

[10] S. Chen and G. D. Doolen. Lattice Boltzmann method for fluid flows. Annual Review of Fluid Mechanics, 30:329-364, 1998.

[11] B. Chopard, A. Dupuis, A. Masselot, and P. Luthi. Cellular automata and lattice Boltzmann techniques: An approach to model and simulate complex systems. $A d-$ vances in Complex Systems, 5(2/3):103-246, 2002.

[12] D. Dab, J.-P. Boon, and Y.-X. Li. Lattice-gas automata for coupled reaction-diffusion equations. Physical Review Letters, 66(19):2535-2538, 1991.

[13] S. P. Dawson, S. Chen, and G. D. Doolen. Lattice Boltzmann computations for reaction-diffusion equations. Journal of Chemical Physics, 98(2):1514-1523, 1993.

[14] G. Dimarco and L. Pareschi. Hybrid multiscale methods I. Hyberbolic relaxation problems. Communications in Mathematical Sciences, 4(1):155-177, 2006.

[15] O. Filippova and D. Hänel. Grid refinement for lattice-BGK models. Journal of Computational Physics, 147:219-228, 1998.

[16] O. Filippova and D. Hänel. Acceleration of lattice-BGK schemes with grid refinement. Journal of Computational Physics, 165:407-427, 2000.

[17] A. L. Garcia, J. B. Bell, W. Y. Crutchfield, and B. J. Alder. Adaptive mesh and algorithm refinement using direct simulation Monte Carlo. Journal of Computational Physics, 154:134-155, 1999.

[18] C. W. Gear, T. J. Kaper, I. G. Kevrekidis, and A. Zagaris. Projecting to a slow manifold: Singularly perturbed systems and legacy codes. SIAM Journal on Applied Dynamical Systems, 4(3):711-732, 2005.

[19] C. W. Gear and I. G. Kevrekidis. Constraint-defined manifolds: A legacy code approach to low-dimensional computation. Journal of Scientific Computing, 25(1):1728, 2005. Also: Technical Report physics/0312094, arXiv e-Print archive.

[20] N. G. Hadjiconstantinou. Hybrid atomistic-continuum formulations and the moving contact-line problem. Journal of Computational Physics, 154:245-265, 1999.

[21] M. Junk. A finite difference interpretation of the lattice Boltzmann method. Numerical Methods for Partial Differential Equations, 17(4):383-402, 2001.

[22] M. Junk, A. Klar, and L.-S. Luo. Asymptotic analysis of the lattice Boltzmann equation. Journal of Computational Physics, 210(2):676-704, 2005.

[23] D. Kandhai, W. Soll, S. Chen, A. Hoekstra, and P. Sloot. Finite-difference latticeBGK methods on nested grids. Computer Physics Communications, 129:100-109, 2000 . 
[24] J. Latt, B. Chopard, and P. Albuquerque. Spatial coupling of a lattice Boltzmann fluid model with a finite difference Navier-Stokes solver. Technical Report physics/0511243, arXiv e-Print archive, November 2005.

[25] P. Le Tallec and F. Mallinger. Coupling Boltzmann and Navier-Stokes equations by half fluxes. Journal of Computational Physics, 136:51-67, 1997.

[26] T. Lee and C.-L. Lin. An Eulerian description of the streaming process in the lattice Boltzmann equation. Journal of Computational Physics, 185:445-471, 2003.

[27] D. J. Mavriplis. Multigrid solution of the steady-state lattice Boltzmann equation. Computers \& Fluids, 35(8-9):793-804, 2006.

[28] R. Mei, L.-S. Luo, P. Lallemand, and D. d'Humières. Consistent initial conditions for lattice Boltzmann simulations. Computers \& Fluids, 35(8/9):855-862, 2006.

[29] Y. H. Qian, D. d'Humières, and P. Lallemand. Lattice BGK models for Navier-Stokes equation. Europhysics Letters, 17(6):479-484, 1992.

[30] Y. H. Qian and S. A. Orszag. Scalings in diffusion-driven reaction $A+B \rightarrow C$ : Numerical simulations by lattice BGK models. Journal of Statistical Physics, 81(1/2):237-253, 1995.

[31] M. Rheinländer. A consistent grid coupling method for lattice-Boltzmann schemes. Journal of Statistical Physics, 121(1-2):49-74, 2005.

[32] J. Tölke, M. Krafczyk, and E. Rank. A multigrid-solver for the discrete Boltzmann equation. Journal of Statistical Physics, 107(1/2):573-591, 2002.

[33] J. Tölke, M. Krafczyk, M. Schulz, and E. Rank. Implicit discretization and nonuniform mesh refinement approaches for FD discretizations of LBGK models. International Journal of Modern Physics C, 9:1143-1155, 1998.

[34] U. Trottenberg, C. Oosterlee, and A. Schüller. Multigrid. Academic Press, 2001.

[35] P. Van Leemput, K. Lust, and I. G. Kevrekidis. Coarse-grained numerical bifurcation analysis of lattice Boltzmann models. Physica D: Nonlinear Phenomena, 210(1-2):58$76,2005$.

[36] P. Van Leemput, W. Vanroose, and D. Roose. Initialization of a lattice Boltzmann model with constrained runs. Technical Report TW 444, K.U.Leuven, Dept. of Computer Science, December 2005. Submitted.

[37] P. Van Leemput, W. Vanroose, and D. Roose. Numerical and analytical spatial coupling of a lattice Boltzmann model and a partial differential equation. In A. Gorban, N. Kazantzis, I. Kevrekidis, H. Ottinger, and C. Theodoropoulos, editors, Model Reduction and Coarse-Graining Approaches for Multiscale Phenomena, pages 423-441. Springer, 2006.

[38] C. Vandekerckhove, P. Van Leemput, and D. Roose. Acceleration of lattice Boltzmann models through state extrapolation: a reaction-diffusion example. Technical Report TW 466, K.U.Leuven, Dept. of Computer Science, August 2006. Submitted.

[39] R. Verberg and A. J. C. Ladd. Simulation of low-Reynolds-number flow via a timeindependent lattice-Boltzmann method. Physical Review E, 60(3):3366-3373, 1999. 